\title{
Morphological aspects of some symmetrical ammonium hydrogen tartrate crystals grown by silica gel technique
}

\author{
C C DESAI and A N HANCHINAL \\ Department of Physics, Sardar Patel University, Vallabh Vidyanagar 388 120, India \\ MS received 30 December 1986; revised 26 February 1987
}

\begin{abstract}
Ammonium hydrogen tartrate single crystals have been prepared by the reaction of $\mathrm{NH}_{4} \mathrm{Cl}$ and tartaric acid in silica gel. The morphology of some as-grown symmetrical crystals has been studied by optical microscopy. The growth morphology studies indicate that the grown crystals are needle-shaped, orthorhombic disphenoidal and tetrahedral disphenoidal. The growth mechanism has been assessed.
\end{abstract}

Keywords. Ammonium hydrogen tartrate; single crystal; gel method; morphology; habit modification.

\section{Introduction}

Crystals show a wide variety of morphology. Questions relating to variations and the relationship of different morphologies to the growth conditions of crystals have been a long and stimulating problem. The relationship between morphologies of crystals and the growth conditions would help in understanding the kinetics processes of the growth mechanism. The morphology and morphology modifications of a variety of crystals have been studied earlier (Burton et al 1951; Mullin 1961; Hartman 1969; Elwell and Scheel 1975).

Ammonium hydrogen tartrate (AHT), $\mathrm{NH}_{4} \mathrm{HC}_{4} \mathrm{H}_{4} \mathrm{O}_{6}$ crystals show many interesting physical properties such as ferroelectricity, piezoelectricity, optical and other characteristics. They are used for controlling laser emission (Pipree and Koblova 1967). The efficiency of AHT crystals in different modulation and defocusing systems is characterized by their electro-optical coefficients. The optimal conditions for the growth of large transparent crystals of AHT in silica gels and the results affecting the nucleation by altering the gel parameters have earlier been reported by Desai and Hanchinal $(1983,1985)$. This paper explains the morphological variations in AHT crystals and its relation to growth conditions.

\section{Experimental}

Single crystals of AHT were grown by the gel technique. The growth process involves the subsequent diffusion of ammonium chloride into a gel in which tartaric acid is diffused before. Due to the slow diffusion of $\mathrm{NH}_{4} \mathrm{Cl}$ in the gel, the reaction region advances gradually into the gel from the gel solution interface. The colourless transparent crystals upto $20 \times 5 \times 3 \mathrm{~mm}^{3}$ in size have been grown in about 2-3 weeks. The crystals were harvested by physical removal from the gel and examined by scanning electron microscope, $x$-ray diffraction, density measurements and thermogravimetric analysis and were confirmed to be AHT. They belong to the orthorhombic crystal 
system and have lattice parameters $a_{0}=0.7640 \pm 0.0002 \mathrm{~nm}, b_{0}=1.3441 \pm 0.0003 \mathrm{~nm}$ and $c_{0}=0.7029 \pm 0.0002 \mathrm{~nm}$ and its space group is $P 22_{1} 2_{1} 2_{1}$, and have 4 molecules per unit cell. The density of AHT is $1.636 \mathrm{~g} \mathrm{~cm}^{-3}$. The crystals do not contain any water molecules as seen from differential scanning calorimetric (DSC) curves. Defect properties of AHT crystals were studied by using a chemical etching technique. A solution of $\mathrm{SrCl}_{2}(1.0 \mathrm{M})$ was used as an etchant which produces well-defined rectangular shaped etch pits at the emergent sites of dislocations on the cleaved (100) face. The dislocation density was found to be $2.1 \times 10^{3} \mathrm{~cm}^{-2}$. The morphology of the crystals has been studied by optical microscopy. The results are based on the statistical average of five sets of experiments.

\section{Results}

In the present investigation, crystals with different morphologies were obtained at different depths in the growing test tubes and at different growth conditions. The morphology of the grown crystals is affected mainly by the concentration of the feed $\left(\mathrm{NH}_{4} \mathrm{Cl}\right)$ solution and the $\mathrm{pH}$ of the system. Three types of morphologies of AHT crystals were observed namely (i) prismatic needle-shaped crystals, (ii) orthorhombic disphenoidal crystals and (iii) disphenoidal tetrahedral crystals.

\subsection{Prismatic needle-shaped crystals}

Usually the nucleation of this type of crystals initiates in the region OA (figure 1a), just below the gel-interface. They start growing from a bunch of nucleii with the diffusion of feed solution into the gel. With lapse of time, the thickness of the crystal increases with faster growth rate along [001] direction, which is the growth direction of AHT crystals of different habits. The growth of needle is mainly due to the presence of diffusion field around the crystal tips of the needle and the crystals grow into the supersaturated solution whereas the sides of the crystals are in contact with a largely exhausted solution. The typical prismatic needle-shaped crystals are illustrated in figure $1 \mathrm{~b}$. The development of the morphology is schematically shown in figure $1 \mathrm{c}$ and it is seen that (100), (110), (130) and (11I) are well-developed faces and the (110) face is fairly developed. The crystals are quite transparent with lusterous faces. These characteristics speak of good quality and perfection of these crystals.

\subsection{Orthorhombic disphenoidal crystals}

In this category, two types of crystals, rod-like orthorhombic and plate-like orthorhombic, are obtained. Fairly high concentrations of $2.0 \mathrm{M}$ to $3.5 \mathrm{M} \mathrm{NH}_{4} \mathrm{Cl}$ feed solutions and a fairly large amount of this solution at $\mathrm{pH}$ between 3 and 4.5 (figure 2a) gave rise to a rod-like orthorhombic disphenoidal type of crystals as shown in figure $2 \mathrm{~b}$, whereas the concentration of feed solution between $0.5 \mathrm{M}$ and $2.0 \mathrm{M}$ at $\mathrm{pH}$ less than 2 (figure $3 a$ ) gave rise to a platelet orthorhombic disphenoidal type of crystals as shown in figure $3 \mathrm{~b}$. The usual regions of crystal growth in the test tubes are clearly illustrated in figures $2 a$ and $3 a$. The morphology of these two types of crystals is depicted in figures $2 \mathrm{c}$ and $3 \mathrm{c}$. 

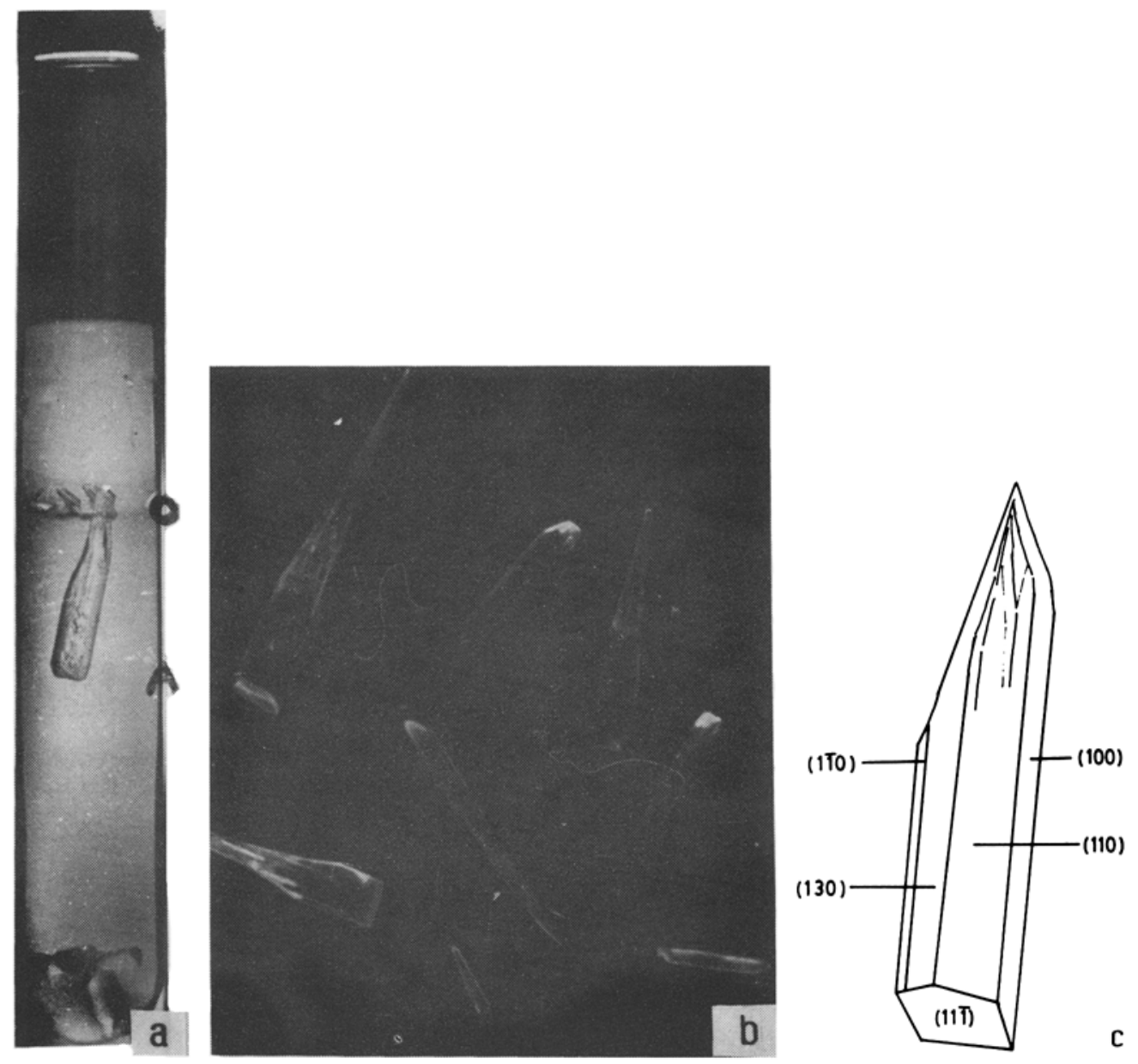

Figure 1. ( $\times 50)$ a. Transparent AHT crystals growing in a silica gel medium. The nucleation initiates in the $O A$ region. b. Some of the typical prismatic needle-shaped crystals. c. Schematic representation of the crystal morphology.

In rod-like disphenoidal crystals the $(100),(110),(11 \overline{1}),(\overline{1} \overline{1} 0)$ and $(\overline{1} \overline{1} 1)$ faces are large and dominating while the (113) and (130) faces are very small. Gel incorporation inclusion is often used in these crystals without affecting their morphology. These inclusions reduce their transparency.

In plate-like disphenoidal crystals, the prominent faces are (100), (111), (111), (2I3), while (110) and (110) faces are not well developed. Most of them are fairly transparent.

\subsection{Disphenoidal tetrahedral crystals}

These crystals usually appear at the top of the interface and at the extreme bottom of the glass test tube as illustrated in figure $4 a$. Within a limited distance above the gel interface. supersaturation takes place because of a spontaneous liberation of liquid 

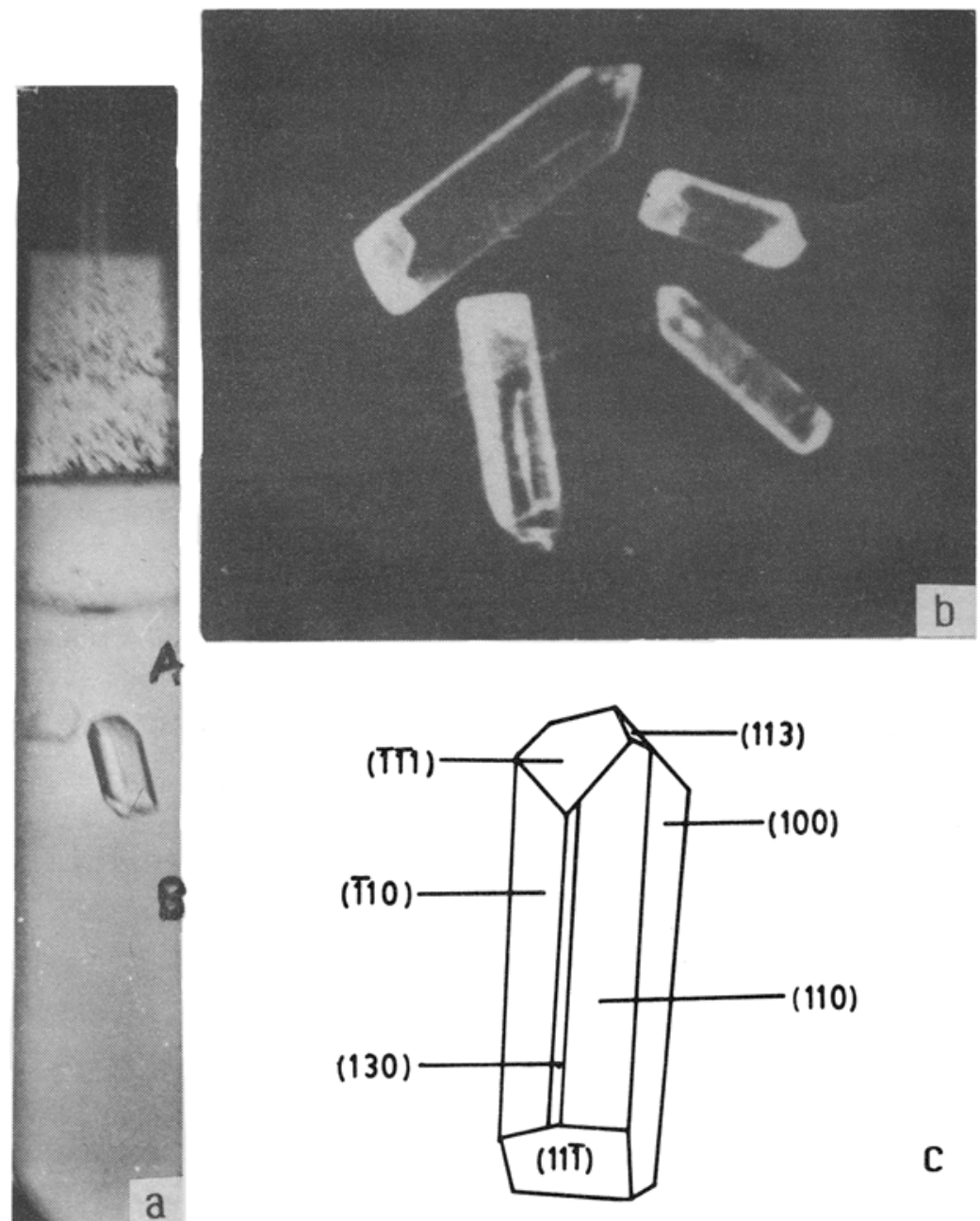

C

Figure 2. $(\times 50)$ a. AHT rod-like orthorhombic disphenoidal crystals growing at $\mathrm{pH} 4$ b. Typical crystals. c. Schematic representation.

from the gel (tartaric acid). This is called syneresis" which is one of the important properties of gels. Syneresis is also considered as an internal debydration. It produces shrinking of the gel thereby changing its pore size. The formation of this type of crystals is believed to be due to lack of place to grow further. At the top of the interface. within a limited distance when nucleation initiates along the [001] directions. the interface perforces to stop the growth. At the bottom, when nucleation takes place within a very short distance from the bottom of the test tubes, the bottom of the tube stops the growth. The limited growth develops only in (111) and (1̄11) faces, and sometimes with slightly devcloped (113) face. A couple of such crystals are illustrated in figure $4 b$ and schematically represented in figure $4 c$. These crystals are transparent.

Occasionally cusps are observed due to the lateral growth of crystals in the test tube. In cusp-like cavities, the gel has been separated from the growing crystals. 


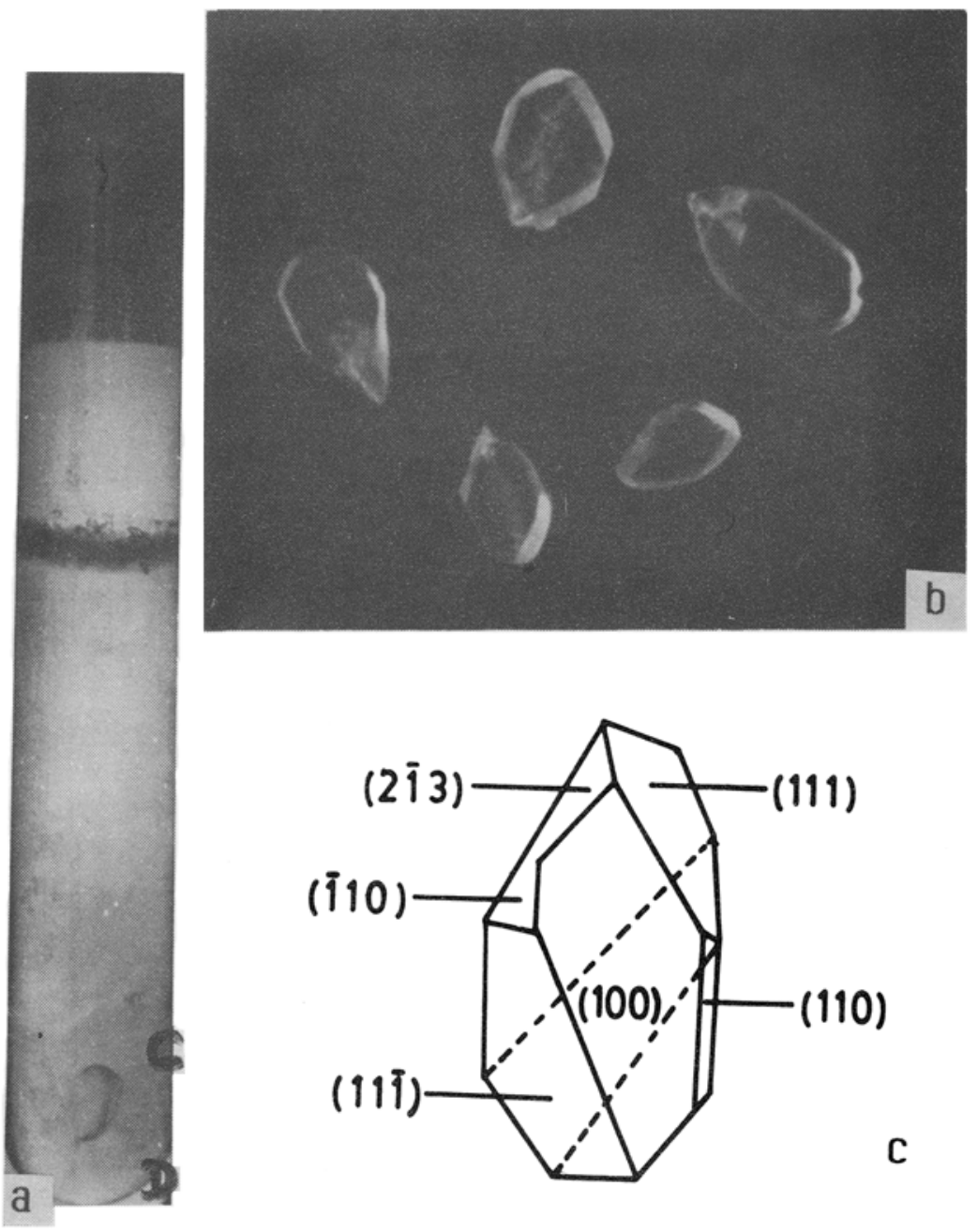

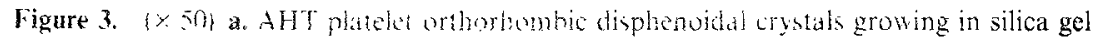
at pH 2 . The nuclestion initiates in CD region. b. Platelet crystals of AHT. c. Schematic representatum of the oryial morpholog?.

These are presumably the outcome of gel displacement from the pressure of advancing growth surface.

The c-axis is the growth axis of AHT crystals. Hence, whenever isolated crystals are grown. irrespective of their orientation with respect to the vertical axis of the test tube, they always grow along ('-axis. In many cases the growth of the axis of isolated crystals is normal to each other. In addition. there are several other factors which influence the morphology and size of the growing crystals stich as gel temperature. gel aging. gel density. gel pH. concentration of feed solution, specific gravity of gel mixture. etc. It is therefore desirable to consider a few of the above factors in details.

\subsection{Influence of gel $\mathrm{pH}$}

The effect of gel pH on the morphology of grown crystals was studied by keeping all 

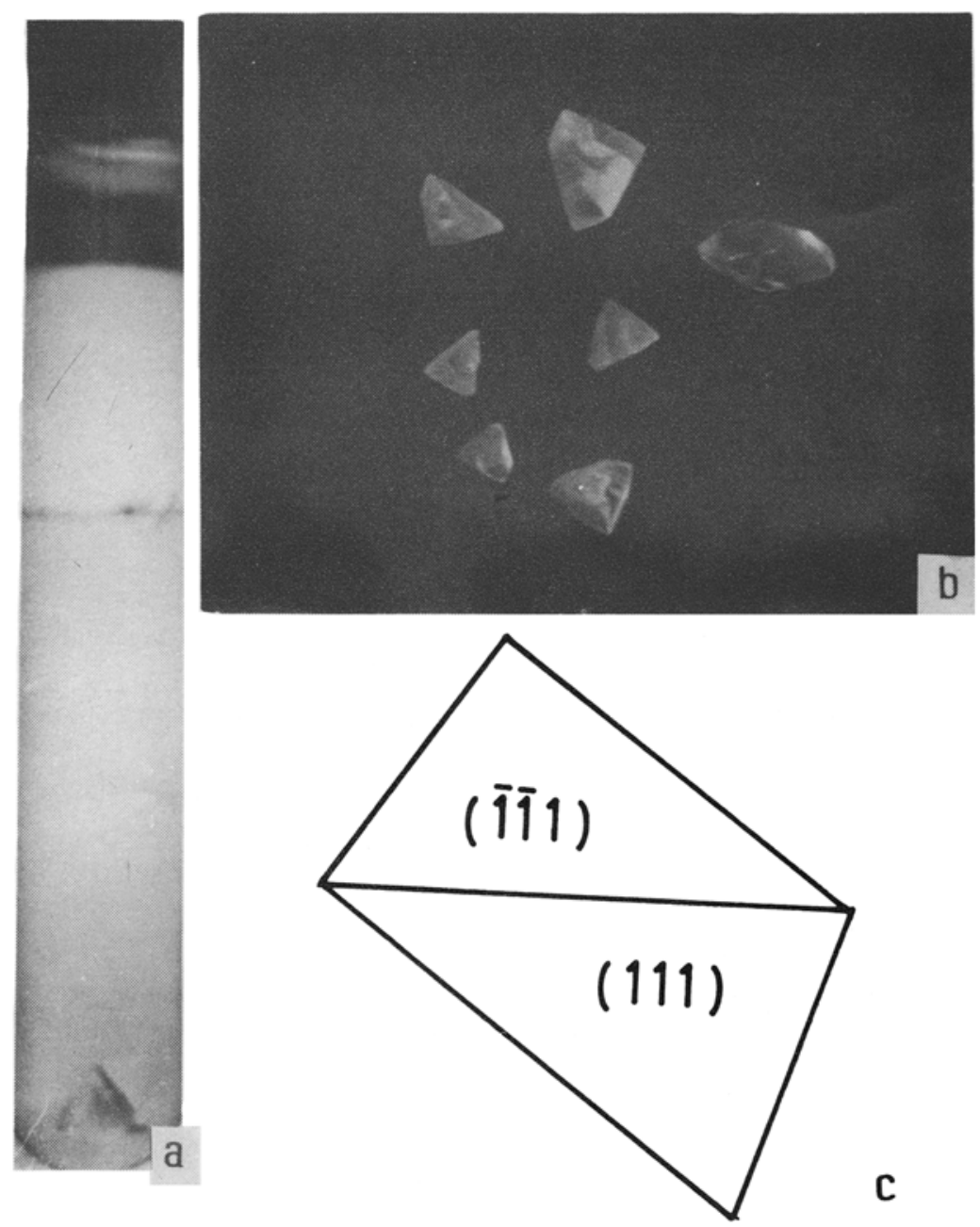

C

Figure 4. 1. 5(1) a. Transparent tetratiedial crystals growing at the bottom of the tube b. Disphenoidal tetrahodal crystals. c. Schematic representation of the tetrahedral crystal morpholigy.

other factors constant. Before gclation, the $\mathrm{pH}$ value of the gel mixture (gel + tartaric acid) was determined by a $\mathrm{pH}$ meter for a known concentration of feed solution. viz $2.0 \mathrm{M}$. The effect of gel $\mathrm{pH}$ was studicd and the results are as follows: $\mathrm{pH}$ range between 4.5 and 7.17 gave rise 10 prismatic needle-shaped crystals. while rod-like orthorhombic disphenoidal crystals obtained between 2.0 and $4.5 \mathrm{pH}$ and $\mathrm{pH}<2.0$ was responsible for the formation of plate-like sphenoidal crystals.

\subsection{Effect of feed solution}

The effect of concentration of feed $\left(\mathrm{NH}_{4} \mathrm{Cl}\right)$ solution on the morphology and size of growing crystals was studied by keeping all other parameters constant and varying the concentration of feed solution. It is observed that increasing the concentration of 
feed solution increases the linear growth along the [001] direction for needle-shaped and disphenoidal crystals. Needle-shaped crystals are translucent and geometrically well-defined. The quality of these crystals deteriorates with an increase in the concentration of feed solution. Disphenoidal crystals are transparent and increase in size with the increase of concentration of feed solution.

\section{Discussion}

Habit modification depends on the phenomenon occurring on crystal surfaces. Diffusion of molecules on the surfaces of growing crystals is an important parameter in this regard. The relative rates of surface diffusion on basal and prism faces are responsible for determining the habit in the very early stages of growth. Crystal habit is governed by the interaction between its surface and the diffusion field. Once the habit has been established, the diffusion field around it will orient itself to conform to the crystal symmetry and tend to maintain it.

The parameters such as concentration of feed solution, $\mathrm{pH}$ of gel, impurities in the solvent, temperature etc have considerable influence on the diffusion rate (or growth rate) which ultimately influences the crystal morphology. In a steady state of concentration gradients, diffusion rate also becomes steady which favour the growth of welldeveloped crystals. However, a relatively slower growth rate along one direction results in the growth of platelet disphenoidal crystals (figure 3a). Preferentially faster growth rate in one particular direction viz $c$-axis, leads to the formation of prismatic needle-shaped crystals (figure 1a). At moderate supersaturation of feed solutions, crystals continue to develop as a tetrahedral disphenoidal crystals (figure 4a). In such cases the excess materials arriving at the edges and corners of the crystals is redistributed over the surface by surface diffusion.

\section{Conclusions}

Transparent prismatic needle-shaped crystals are obtained. Platelets and rod-like orthorhombic and tetrahedral sphenoidal crystals are obtained under different growth conditions. Increasing concentration of feed solutions increases the linear growth along [001] direction for needle-shaped and disphenoidal crystals. Generally crystals are transparent, but opaque crystals result in the event of gel inclusion. Restricted amount of gel inclusion takes place in rod-like disphenoidal crystals. Gels set with tartaric acid give better crystals than those set with mineral acids.

\section{Acknowledgements}

One of the authors (CCD) is grateful to the University Grants Commission, New Delhi, for financial assistance. Thanks are also due to Dr K C Desai and Mrs Lila K Desai (Belleville, New Jersey) for their interest and encouragement.

\section{References}

Burton W K, Cabrera N and Frank F C 1951 Philos. Trans. R. Soc. London A243 299

Desai C C and Hanchinal A N 1983 Cryst. Res. Technol. 181499 
Desai C C and Hanchinal A N 1985 Surface Technol. 2569

Elwell D and Scheel H J 1975 Crystal growth from high temperature solutions (London: Academic Press)

Hartman P 1969 Growth of crystals (ed.) N N Shiftal (New York: Consultants Bureau) p. 8

Mullin J W 1961 Crystallization (London: Butterwortbs)

Pipree L V and Koblova M M 1967 Radio Eng. Electron. Phys. (Engl. Transl.) 12503 